\title{
BMJ Open Effect of the rate of chest compression familiarised in previous training on the depth of chest compression during metronome-guided cardiopulmonary resuscitation: a randomised crossover trial
}

\author{
Jinkun Bae, ${ }^{1}$ Tae Nyoung Chung, ${ }^{1}$ Sang Mo Je ${ }^{2}$
}

To cite: Bae J, Chung TN, Je SM. Effect of the rate of chest compression familiarised in previous training on the depth of chest compression during metronome-guided cardiopulmonary resuscitation: a randomised crossover trial. BMJ Open 2016;6: 010873 . doi:10.1136/bmjopen-2015010873

- Prepublication history for this paper is available online. To view these files please visit the journal online (http://dx.doi.org/10.1136/ bmjopen-2015-010873).

Received 14 December 2015 Revised 18 January 2016 Accepted 26 January 2016

CrossMark

\section{${ }^{1}$ Department of Emergency Medicine, CHA University School of Medicine, Gyeonggi-Do, Republic of Korea \\ ${ }^{2}$ Department of Pediatrics, CHA University School of Medicine, Gyeonggi-Do, Republic of Korea}

Correspondence to Dr Tae Nyoung Chung; hendrix74@gmail.com

\section{ABSTRACT}

Objectives: To assess how the quality of metronomeguided cardiopulmonary resuscitation (CPR) was affected by the chest compression rate familiarised by training before the performance and to determine a possible mechanism for any effect shown.

Design: Prospective crossover trial of a simulated, one-person, chest-compression-only CPR.

Setting: Participants were recruited from a medical school and two paramedic schools of South Korea.

Participants: 42 senior students of a medical school and two paramedic schools were enrolled but five dropped out due to physical restraints.

Intervention: Senior medical and paramedic students performed 1 min of metronome-guided CPR with chest compressions only at a speed of 120 compressions/ min after training for chest compression with three different rates (100, 120 and 140 compressions/min). Friedman's test was used to compare average compression depths based on the different rates used during training.

Results: Average compression depths were significantly different according to the rate used in training $(p<0.001)$. A post hoc analysis showed that average compression depths were significantly different between trials after training at a speed of 100 compressions/min and those at speeds of 120 and 140 compressions/min (both $\mathrm{p}<0.001$ ).

Conclusions: The depth of chest compression during metronome-guided CPR is affected by the relative difference between the rate of metronome guidance and the chest compression rate practised in previous training.

\section{INTRODUCTION}

Results of recent clinical studies which showed a significant relationship between chest compression rate and survival after

\section{Strengths and limitations of this study}

This is the first study to assess directly how the rate of chest compression used in previous training affects the quality of metronome-guided cardiopulmonary resuscitation (CPR).

- Our study suggests the necessity for considering the training status of rescuers regarding chest compression rate before planning metronomeguided CPR.

- Our results provide a clue to explain the discordance in the results of prior research assessing the effect of the rate of guiding sound on the quality of metronome-guided CPR.

- Our results were derived from a simulated trial of chest compression only CPR, which may not represent all aspects of a real clinical setting.

out-of-hospital cardiac arrest suggested the necessity for keeping the rate of chest compressions between 100 and $120 / \mathrm{min}^{1}{ }^{{ }^{2}}$ Metronome guidance may be a good assistance to keep chest compression rate at this relatively narrow target range, considering the prior evidence which showed its efficacy in keeping chest compression rate without compromising other quality components of cardiopulmonary resuscitation (CPR). ${ }^{3-6}$

Performance of chest compression following metronome guidance at a certain rate may be a slowdown of chest compression for a rescuer who is familiarised with faster chest compression by training, while it may be a speed-up of chest compression for a rescuer who is familiarised with slower chest compression. Limitation of chest compression speed may limit its depth, considering the linear correlation of chest compression rate and depth shown in a prior study performed under an experimental condition in which the effect of 
rescuer fatigue could relatively be excluded. ${ }^{7}$ However, none of the prior researches studying the usefulness of a metronome for assisting CPR specifically assessed how a baseline training status regarding chest compression rate affected the quality of metronome-guided CPR. We hypothesised that induction of chest compression at a certain rate during metronome-guided CPR would decrease its depth if a rescuer was familiarised with faster chest compression in previous training, while it would increase the depth if he (or she) was familiarised with slower chest compression. To test the hypothesis, we aimed to assess how the quality of metronome-guided CPR was affected by the change of chest compression rate used in training. We also aimed to determine a possible mechanism for any such difference regarding critical quality factors such as the depth of chest compression.

\section{METHODS}

This study was performed under the approval of the CHA University Bundang Medical Center Institutional Review Board (IRB approval \# BD2014-019, 2 February 2014). This was a single-blinded, randomised, prospective crossover trial of simulated, one-person, chest-compression-only CPR.

The study was performed at the CHA University Bundang Medical Center, Gyeonggi-Do, Korea, from March 2014 to February 2015. We enrolled senior students of a medical school (CHA University) and two paramedic schools (Seojeong College and Eulji University) who, within the previous year, had finished two sessions of regular CPR training courses based on the American Heart Association (AHA) Basic Life Support course according to the 2010 AHA guidelines for CPR and emergency cardiovascular care. ${ }^{8}$ Any student who declined to participate in the study for any reason was excluded. Written informed consent was obtained from every participant.

A ResusciAnne manikin with a PC skill-reporting system (Laerdal, Stavanger, Norway) was used to measure and record CPR data. Chest compression guiding sounds at speeds of 100, 120 and 140 compressions/min were synthesised using Reason audio synthesis software (Propellerhead, Stockholm, Sweden).

The experimental procedure consisted of three trials with two phases: a training phase for the participants to become accustomed to a certain chest compression rate, and a measuring phase to measure the quality of chest compressions while performing CPR following metronome guidance at a conventional rate of chest compression $(120 / \mathrm{min})$. The participants practised chest-compression-only CPR following the metronomeguided sound at a rate randomly selected from 100, 120 or 140 compressions/min for $2 \mathrm{~h}$ as a training phase. Randomised selection of a rate was based on a random number table generated by Microsoft Office Excel 2010 (Redmond, Washington, USA). The rate and the depth of chest compression without metronome guidance were measured for $1 \mathrm{~min}$ shortly after the practice. After $1 \mathrm{~h}$ of rest, the participants performed chest compression-only CPR for $1 \mathrm{~min}$ following the metronome-guided sound at a rate of 120 compressions/ min, and their quality was measured. This was the first trial. As the training phase for the second trial, the participants again performed chest compression-only CPR following the metronome-guided sound at a rate randomly selected between the two not selected in the first trial. Then the rest of the trial was repeated. For the third trial, a training phase was performed using the guiding sound at the rate unselected in the prior two trials. The duration of the interval between each trial was guaranteed to be least 2 days to prevent a possible interaction of different chest compression rates practised in each training phase (figure 1). Only one participant was included at a time in each trial. The rate of metronome guidance used in every trial was blinded to the participants.

The sex, age, body weight and height of the participants were collected. Average compression depth (ACD, $\mathrm{mm}$ ), average compression rate (ACR, counts/min), duty cycle $(\%)$, and the fractions of chest compressions with incomplete release and incorrect hand position (\%) were measured and recorded during the measuring phase of the procedure using the PC skill-reporting system.

The data following a normal distribution were described as mean $\pm \mathrm{SD}$, and non-parametric data were described as median (IQR). A repeated measures analysis of variance (ANOVA) was conducted to compare normally distributed variables, and a paired t-test with a Bonferroni correction was performed as a post hoc test. A Friedman test was used to compare non-normally distributed variables, and a Wilcoxon signed rank test with Bonferroni correction was performed as a post hoc test. Statistical significance was set at $\mathrm{p}<0.05$, which was calculated from a two-sided test, if applicable. A sample size of 32 was required to have sufficient power to detect a significant difference in the ACD of each trial using repeated measure ANOVA (effect size $\mathrm{f}=0.25$, power $=0.80$ ). Microsoft Office Excel 2010 was used to record and analyse data, and IBM SPSS Statistics 21.0 (IBM Corp., Armonk, New York, USA) was used for statistical calculations. R V.3.1.3 (R-project, http://www. r-project.org) was used to build a parallel-coordinates plot and a box plot of the between-group difference for visualising the results of Friedman's test. G*Power 3.1 (Heinlich-Heine Universität, Düsseldorf, Germany) was used to calculate the sample size. $^{9}$

\section{RESULTS}

A total of 42 senior medical and paramedic students enrolled in the study. Five participants dropped out during the performance due to physical restraint in the training phase, so data from 37 students were included in the analysis. The baseline data of the participants are summarised in table 1 .

There was a significant difference in ACD in the measuring phase according to the rate of chest compression 


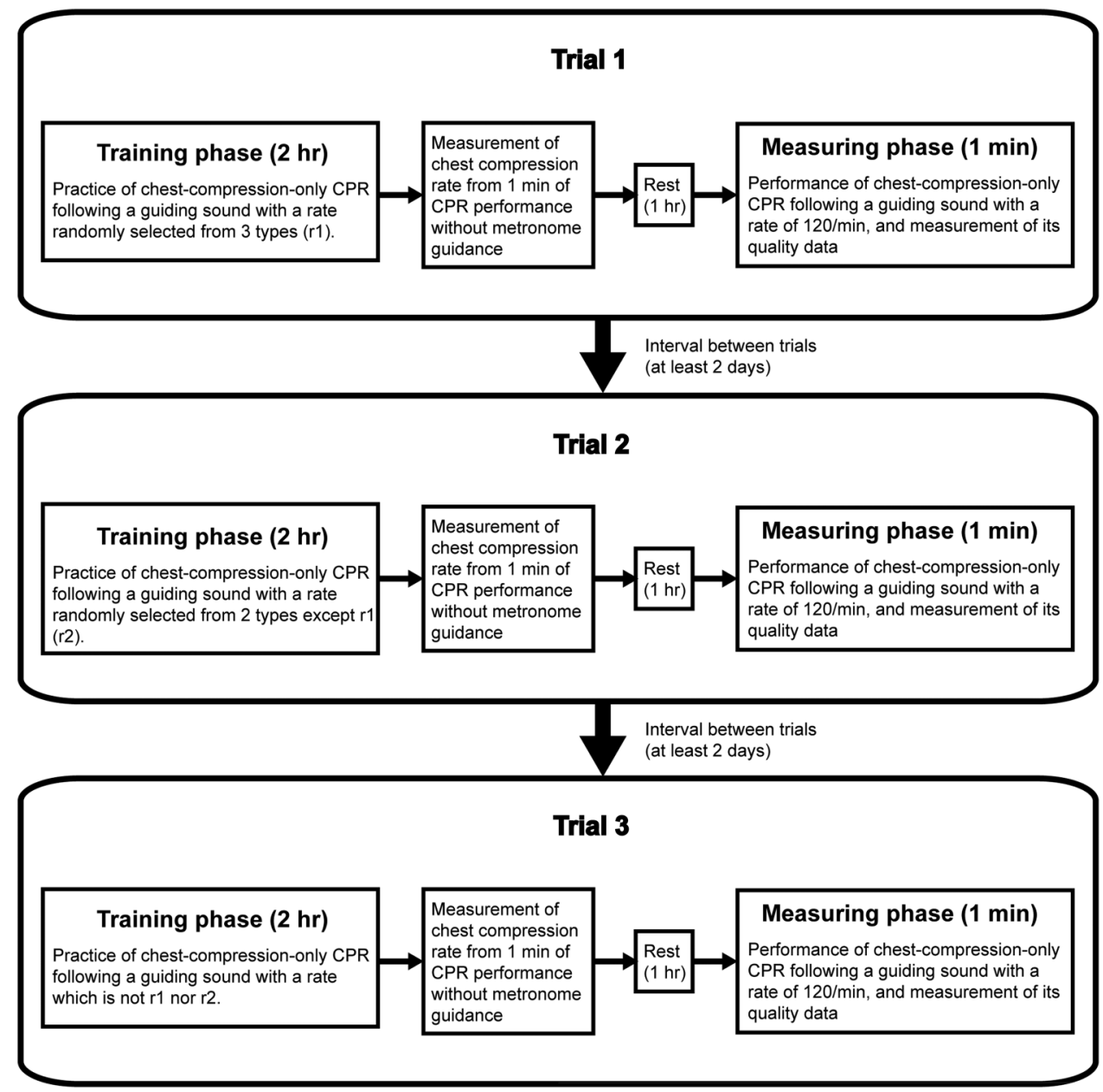

Figure 1 Flow diagram of the experimental procedure. CPR, cardiopulmonary resuscitation.

used in a training phase which was 100,120 or $140 / \mathrm{min}$ $(\mathrm{ACD}=55.0 \quad(50.0-60.0), \quad 53.0 \quad(45.5-57.5)$, and 51.0 (43.5-59.0), mm, respectively) $(\mathrm{p}<0.001)$ (figure $2 \mathrm{~A})$. Post hoc analysis revealed the differences between the trials with a rate of $100 / \mathrm{min}$ used in a training phase (t100) and those with a rate of $120 / \mathrm{min}$ (t120) $(\mathrm{p}<0.001)$, and between $\mathrm{t} 100$ and those with a rate of $140 / \min (\mathrm{t} 140)(\mathrm{p}=0.001)$. There was no significant difference between t120 and t140 ( $\mathrm{p}=0.517)$ (figure 2B).

There was also a significant difference of duty cycle among t100, t120 and t140 (duty cycle=37.0 (33.5-39.0),

\begin{tabular}{lc} 
Table 1 Characteristics of the participants \\
\hline Age (year) & $26(24-28)$ \\
Sex (count, \%) & \\
$\quad$ Male & $19,51.4$ \\
$\quad$ Female & $18,41.6$ \\
Background (count, \%) & \\
$\quad$ Medical & $26,70.3$ \\
$\quad$ Paramedic & $11,29.7$ \\
Weight (kg) & $59.4 \pm 11.6$ \\
Height (cm) & $168.0 \pm 7.7$ \\
\hline Age was described median (IQR). Weight and height were \\
described as mean \pm SD.
\end{tabular}

40.0 (37.0-43.5) and 40.0 (38.0-42.5), \%, respectively) $(\mathrm{p}<0.001)$ (figure 3A). Post hoc analysis revealed differences between $\mathrm{t} 100$ and $\mathrm{t} 120 \quad(\mathrm{p}<0.001)$ and between $\mathrm{t} 100$ and $\mathrm{t} 140(\mathrm{p}<0.001)$. There was no significant difference between $\mathrm{t} 120$ and $\mathrm{t} 140 \quad(\mathrm{p}=0.801)$ (figure 3B).

The rate of chest compression measured shortly after the training phase was significantly different according to the rate of metronome guidance used (rate $=96.9 \pm 8.3$, $125.7 \pm 9.6$, and $142.4 \pm 7.4$ compressions/min, respectively) $(\mathrm{p}<0.001)$, with significant post hoc test results ( $\mathrm{p}<0.001$ for all comparisons), while there was no significant difference in the depth $(\mathrm{ACD}=55.0$ (49.5-59.0), 55.0 (43.5-59.0), and 53.0 (43.5-59.0), $\mathrm{mm}$, respectively) $(\mathrm{p}=0.063)$. There was no significant difference in the ratio of incomplete release or improper hand position in the measuring phase according to the rate used in the training phase (both $\mathrm{p}=0.232$ and 0.368 ).

\section{DISCUSSION}

This is the first study to assess directly how the rate of chest compression used in previous training affects the quality of metronome-guided CPR. Our results suggest that the relative difference in the rate of chest 

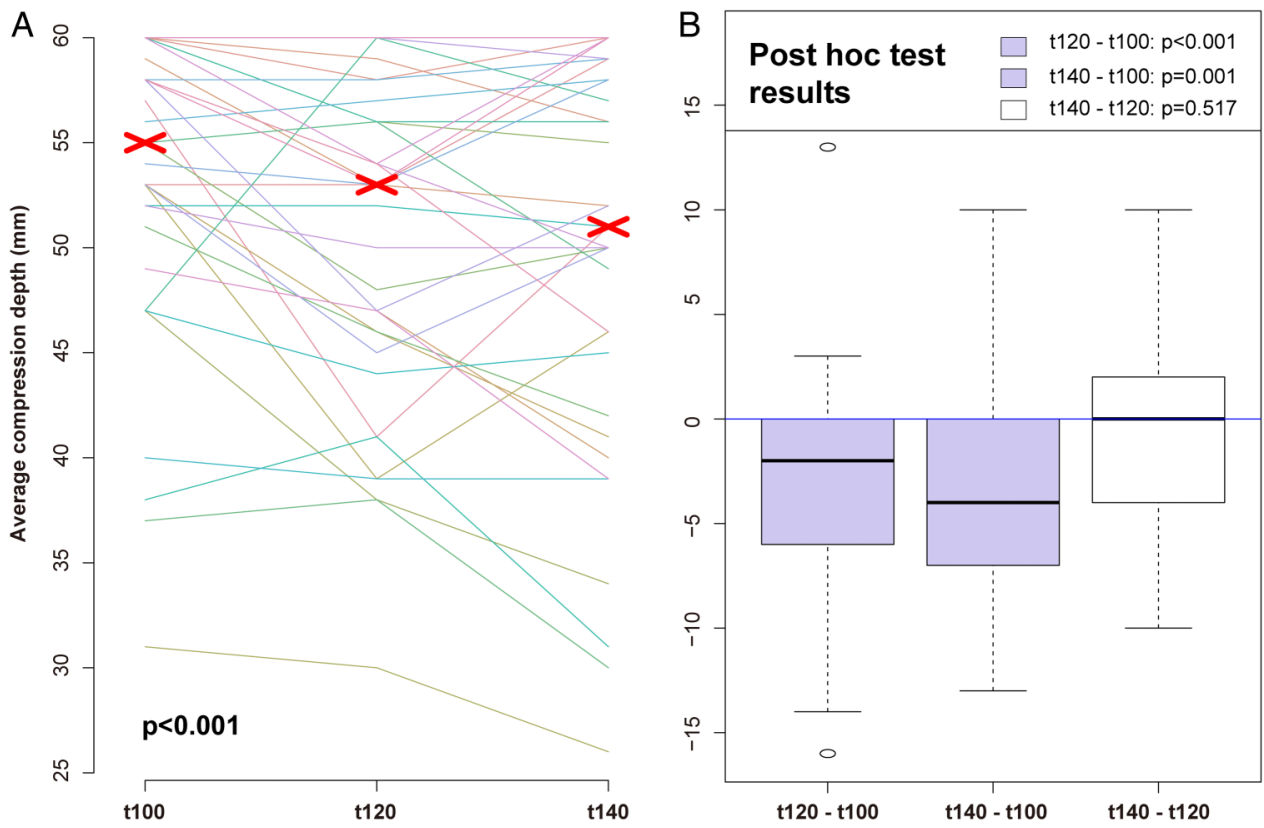

Figure 2 (A) Parallel coordinates plot of average compression depths according to the rate of chest compressions used in training. A red $\mathrm{X}$ symbol represents a median of average compression depths in each trial. $\mathrm{p}$ value was calculated from a Friedman test. t100: the trials with a rate of 100 compressions/min used in training, t120: the trials with a rate of 120 compressions/min used in training, $\mathrm{t} 140$ : the trials with a rate of 140 compressions/min used in training. (B) Box plot of the between-group difference in average compression depths according to the rate of chest compressions used in training. $p$ Values were calculated from Wilcoxon signed rank tests. A significant level of $p$ value was adjusted to below 0.017 after Bonferroni correction.

compression familiarised in practice affects the depth of chest compression when CPR is performed following metronome guidance at a certain rate.
We observed a significant difference in ACD in the measuring phase according to the rate practised in the training phase and showed that the depth of chest
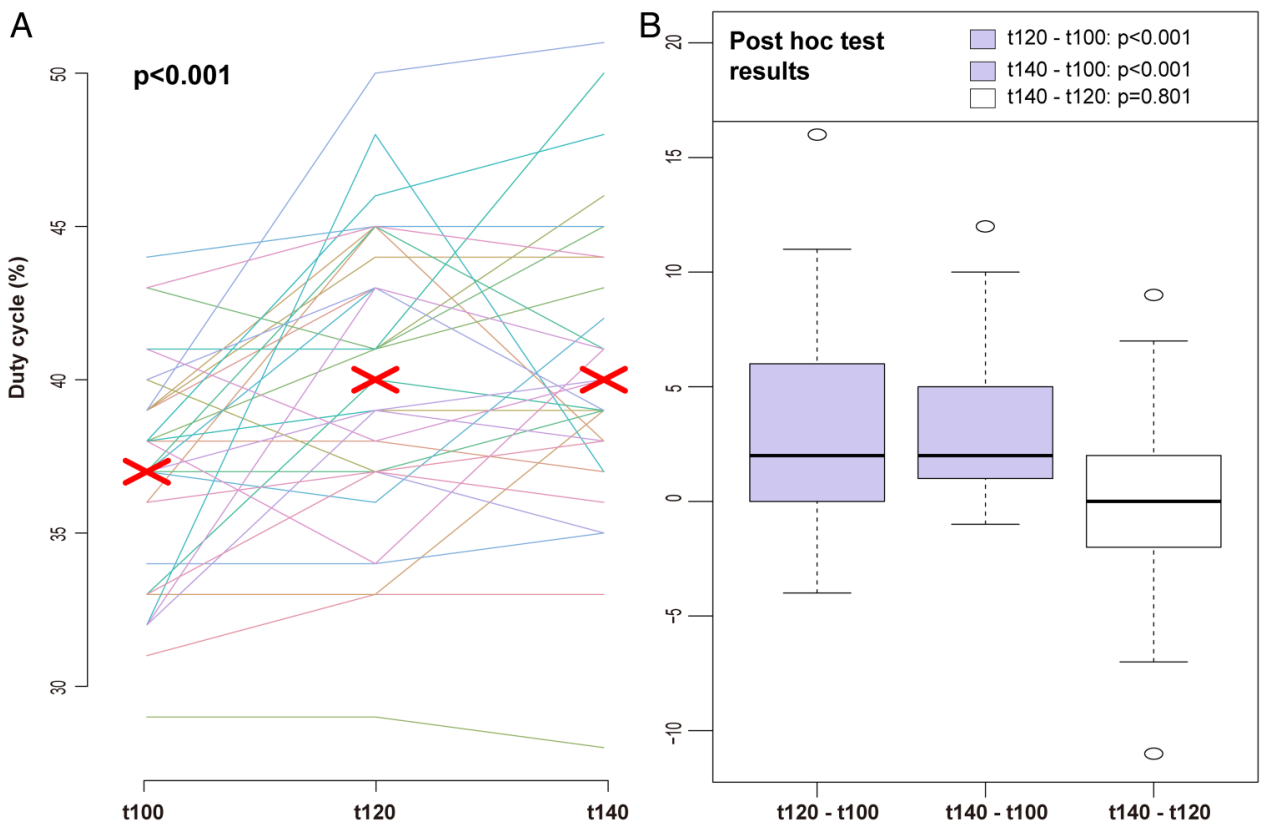

Figure 3 (A) Parallel coordinates plot of duty cycles according to the rate of chest compressions used in training. The red $X$ symbol represents a median of duty cycles in each trial. $p$ value was calculated from a Friedman test. 1100 : the trials with a rate of 100 compressions/min used in training, $\mathrm{t} 120$ : the trials with a rate of 120 compressions/min used in training, $\mathrm{t} 140$ : the trials with a rate of 140 compressions/min used in training. (B) Box plot of the between-group difference in duty cycles according to the rate of chest compressions used in training. $p$ values were calculated from Wilcoxon signed rank tests. A significant level of $\mathrm{p}$ value was adjusted to below 0.017 after Bonferroni correction. 
compression, which is one of the most important quality factors of CPR, can differ based on the rate of chest compression trained in practice, even under the guidance of an identical metronome sound. Moreover, the results of our post hoc analysis showed that deeper chest compression was induced when the rate of metronomeguided sound was faster than in the training phase. This finding, which is that limitation of chest compression speed at a rate equal to or below that familiarised by training may limit its depth, supports our hypothesis, and suggests that the consideration for training status of rescuers regarding chest compression rate is quite necessary before planning metronome-guided CPR. This may also provide a clue to explain the discordance in the results of prior research assessing the effect of the rate of guiding sound on the quality of metronome-guided CPR. ${ }^{7}{ }^{10} 11$ Difference in the trained status of the studied population regarding the practised rate of chest compression may be a cause of the discordance, because none of those researches had considered it.

The duty cycle in the measuring phase was significantly shorter when the rate of chest compression in the training phase was slower than the rate of metronome guidance in the measuring phase. This trend regarding the duty cycle is similar but in an opposite direction to those regarding ACD, suggesting that the difference in chest compression depth according to the pre-trained rate, shown in our experiment, might be related to the duty cycle of chest compression. We think that limitation of chest compression speed also causes a longer duty cycle, resulting in a lower depth, based on a prior study that showed an inverse correlation of compression fraction and chest compression depth. ${ }^{12}$ This effect of the training status regarding the rate of chest compression on the depth may be more critical if CPR is performed by a female or lightweight rescuer, considering the results of another study which showed that the inverse correlation of the duty cycle and ACD was more prominent in those groups. ${ }^{13}$

The results of our analysis of chest compression rates measured just after the training phase showed that our experimental intervention to make the participants accustomed to a certain rate of chest compression worked successfully. Additionally, the results regarding the ratios of incomplete release or improper hand position showed that alteration of the rate of chest compression in the training phase did not affect the error rate of chest compression performance in the measuring phase. These results confirmed that our experiment was performed as intended.

According to our results, deeper chest compression is induced during metronome-guided CPR if the rate of metronome guidance is faster than in practice. This may be interpreted as that the use of a slower chest compression rate in training, even more than that recommended in the guidelines for CPR, is better to acquire higher chest compression depth in the real practice of metronome-guided CPR, which is expected to use the rate recommended in the guidelines. However, great caution is necessary to translate our results in this manner, because our experiment was only designed to assess how the baseline training status of the participants regarding chest compression rate affects the quality of metronome-guided CPR. Rather, the results would better be understood as that metronome-guided CPR without any consideration for a trained chest compression rate of rescuers can be useless according to circumstances.

Our study had a few limitations. First, our results were based on data measured from simulated chest compression-only CPR. Furthermore, we adopted some experimental conditions which might be different from real practice. Hence, great caution is needed before implementing our results in clinical settings. Second, our study was designed as a crossover trial. Subsequently, there could be an interaction caused by the different rate of chest compression trained in each trial. However, we tried to overcome this limitation by placing enough intervals (more than 2 days) between each trial and full randomisation of the sequence of the trials. Moreover, we performed the analysis of chest compression rate data measured just after the training phase without metronome guidance to rule out the possibility of that interaction. Third, we did not consider the rate of chest compression used in previous CPR training courses which were requisite for enrolment to the study. Intervals between those courses and the study performances were not considered either, which might be different from each other. These facts could cause bias of the results. However, the effects were thought to be minimal, if there had been any, considering the result from the comparison of chest compression quality data acquired shortly after the training phase. Furthermore, this was an important reason why we chose a crossover design, which makes baseline conditions other than intended intervention equal for each comparing group.

\section{CONCLUSION}

The depth of chest compression during metronomeguided CPR is affected by the relative difference between the rate of metronome guidance and chest compression rate practised in training. Limiting chest compression rate equal to or below that familiarised in training may lower chest compression depth during metronome-guided CPR.

Contributors All three authors have made substantial contributions to all of the following: (1) the conception and design of the study, or acquisition of data, or analysis and interpretation of data, (2) drafting the article or revising it critically for important intellectual content, (3) final approval of the version to be submitted. In detail: JB was involved in the acquisition of data, analysis and interpretation of data, drafting of the manuscript, final approval given; TNC was involved in the conception, design, analysis and interpretation of data, drafting of the manuscript, final approval given; SMJ was involved in the acquisition of data, critical revision of manuscript, final approval given. 
Funding This research received no specific grant from any funding agency in the public, commercial or not-for-profit sectors.

Competing interests None declared.

Ethics approval Institutional review board of the CHA University Bundang Medical Center.

Provenance and peer review Not commissioned; externally peer reviewed.

Data sharing statement No additional data are available.

Open Access This is an Open Access article distributed in accordance with the Creative Commons Attribution Non Commercial (CC BY-NC 4.0) license, which permits others to distribute, remix, adapt, build upon this work noncommercially, and license their derivative works on different terms, provided the original work is properly cited and the use is non-commercial. See: http:// creativecommons.org/licenses/by-nc/4.0/

\section{REFERENCES}

1. Idris $A H$, Guffey D, Aufderheide TP, et al. Relationship between chest compression rates and outcomes from cardiac arrest. Circulation 2012;125:3004-12.

2. Idris AH, Guffey D, Pepe PE, et al. Chest compression rates and survival following out-of-hospital cardiac arrest. Crit Care Med 2015;43:840-8.

3. Berg RA, Sanders AB, Milander M, et al. Efficacy of audio-prompted rate guidance in improving resuscitator performance of cardiopulmonary resuscitation on children. Acad Emerg Med 1994;1:35-40.
4. Chung TN, Kim SW, You JS, et al. The specific effect of metronome guidance on the quality of one-person cardiopulmonary resuscitation and rescuer fatigue. J Emerg Med 2012;43:1049-54.

5. Milander MM, Hiscok PS, Sanders AB, et al. Chest compression and ventilation rates during cardiopulmonary resuscitation: the effects of audible tone guidance. Acad Emerg Med 1995;2:708-13.

6. Woollard M, Poposki J, McWhinnie B, et al. Achy breaky makey wakey heart? A randomised crossover trial of musical prompts. Emerg Med J 2012;29:290-4.

7. Chung TN, Kim SW, You JS, et al. A higher chest compression rate may be necessary for metronome-guided cardiopulmonary resuscitation. Am J Emerg Med 2012;30:226-30.

8. Jauch EC, Cucchiara B, Adeoye O, et al. Part 11: adult stroke: 2010 American Heart Association Guidelines for Cardiopulmonary Resuscitation and Emergency Cardiovascular Care. Circulation 2010;122(Suppl 3):S818-28.

9. Faul F, Erdfelder E, Lang AG, et al. ${ }^{\star}{ }^{\star}$ Power 3: a flexible statistical power analysis program for the social, behavioral, and biomedical sciences. Behav Res Methods 2007;39:175-91.

10. Field RA, Soar J, Davies RP, et al. The impact of chest compression rates on quality of chest compressions - a manikin study. Resuscitation 2012;83:360-4.

11. Lee $\mathrm{SH}, \mathrm{Kim} \mathrm{K}$, Lee $\mathrm{JH}$, et al. Does the quality of chest compressions deteriorate when the chest compression rate is above 120/min? Emerg Med J 2014;31:645-8.

12. Chung TN, Bae J, Kim EC, et al. Induction of a shorter compression phase is correlated with a deeper chest compression during metronome-guided cardiopulmonary resuscitation: a manikin study. Emerg Med J 2013;30:551-4.

13. Lee CJ, Chung TN, Bae J, et al. $50 \%$ duty cycle May be inappropriate to achieve a sufficient chest compression depth when cardiopulmonary resuscitation is performed by female or light rescuers. Clin Exp Emerg Med 2015;2:9-15. 